\title{
Pervasive Health Monitoring Based on Internet of Things: Two Case Studies
}

\author{
Anurag ${ }^{1}$, Sanaz Rahimi Moosavi ${ }^{1}$, Amir-Mohammad Rahmani ${ }^{1}$, Tomi Westerlund ${ }^{1}$, Geng Yang $^{2}$, \\ Pasi Liljeberg ${ }^{1}$, and Hannu Tenhunen ${ }^{1}$ \\ ${ }^{1}$ Department of Information Technology, University of Turku, Turku, Finland \\ ${ }^{2}$ School of Information and Communication, Royal Institute of Technology (KTH), Stockholm, Sweden \\ Email: \{anutha, saramo, amirah, tovewe, pakrli, hatenhu\}@utu.fi, gengy@kth.se
}

\begin{abstract}
With the continuous evolution of wireless sensor networks and Internet of Things (IoT) various aspects of life will benefit. IoT based pervasive healthcare system has potential to provide error free medical data and alerting system in critical conditions with continuous monitoring. The system will minimize the need of dedicated medical personnel for patient monitoring and help the patients to lead a normal life besides providing them with high quality medical service. In this paper, we provide the implementation of IoT-based architectures for remote health monitoring based on two popular wireless technologies, Wi-Fi and ZigBee. We analyse the two architectures with the aim of identifying their pros and cons and discuss suitability of mentioned wireless communication technologies for different healthcare application domains.
\end{abstract}

Abstract-Internet of Things, e-Health, ZigBee , Wi-Fi, Wireless Sensor Network (WSN), Remote Patient Monitoring.

\section{INTRODUCTION}

Internet of Things (IoT) is enabling and revolutionising the way in which physical objects are communicating with each other. IoT can be utilised in several application domains such as: smart homes and cities, food safety and security and healthcare. The possibilities that IoT provides will innovate novel applications and devices whose communication capability will create new markets and a new economy. It is predicted that number of devices with the internet capability (connected to internet) will be around 25 billion by 2015 and 50 billion by 2020 [1].

IoT offers enormous opportunities to revolutionise healthcare in the near future. It can play a vital role in a wide range of healthcare devices that, for example, enable remote vital sign monitoring in hospitals and more importantly at home. Indeed, remote monitoring offers tremendous possibilities to decrease the costs of healthcare, and, at the same time, to increase healthcare quality by identifying and preventing diseases. In many cases health care is becoming increasingly costly, as patients are required to stay in hospital for the entire duration of their treatment due to the lack of devices with a capability to remotely provide patient's health information to authorised health professionals. Using IoT, gathering patient's health information and transferring it in real time to healthcare professionals will not only reduce the cost of healthcare services but also enable the treatment of health issues before they become critical.

In this paper, we present a health monitoring wireless sensor network architecture and assess the usability of two wireless communication technologies in the presented context. The aim is to identify the advantages and shortcomings of these architectures and find application domains in which these architectures can be properly utilized.

There exist several wireless communication technologies such as Bluetooth, ZigBee, 6LoWPAN or Wi-Fi that can be used to implement wireless network systems. Every technology has its own advantages and drawbacks. The most suitable technology strongly depends on the application requirements. For our health monitoring platform, we use Wi-Fi and ZigBee wireless technologies. For example, if a ZigBee based sensor network is supposed to transfer data to smart phones or tablets, which normally does not support IEEE 802.15.4 standard, a translation gateway is needed to transform ZigBee to another protocol such as Wi-Fi or Bluetooth. To avoid transforming protocols, interoperability should be an intrinsic feature of a sensor based wireless network. For this purpose, Wi-Fi is one of the most popular choices for wireless communication protocol.

\section{RELATED WORK AND MOTIVATION}

There have been many efforts in the field of IoT based remote patient monitoring systems. Piccini et al. [2] discuss wireless system based on Bluetooth for acquiring bio-medical signals such as Electrocardiography (ECG), Electromyography (EMG), Electroencephalography (EEG) and Electrooculography (EOG). The architecture consists of two operational units: one to acquire single lead ECG signal and the other a DSP system to clean the acquired signal from the first unit. More research is required for integrating the associated sensors with a hardware board and miniaturising the system to make it wearable. She et al. [3] present a wireless sensor network architecture based on the IEEE 802.15.4 standard (ZigBee) and $3 \mathrm{G}$ networks for healthcare applications for home or hospital. The system reads signals including ECG, EMG, EEG and EOG, heart rate, breathing and blood pressure, processes it and sends it to a remote server or displays it over LCD screen. The system implements priority scheduling and data compression, which reduces the transmission delays of critical signals and saves bandwidth and power. Lo et al. [4] explain body sensor network (BSN) based on the IEEE 802.15.4 standard which not only monitors and process medical data such as ECG and $\mathrm{SpO} 2$ but also implements context aware sensing with the help of context sensors (e.g. temperature, accelerometer, and humidity). The BSN is power efficient requiring only $0.01 \mathrm{~mA}$ in active mode and $1.3 \mathrm{~mA}$ for computations such as fast Fourier transform (FFT). The collected and processed data is displayed by a flash BSN card for PDAs. A PDA also works as an access point to send the processed data to a central server. Istepanian et al. [5] propose m-IoT (Internet of M-Health Things), an IP based wireless sensor network architecture based on 6LoWPAN, which is used to 
measure medical data such as glucose level in blood and blood pressure. A central access point collects data from the sensor nodes and send to IP based medical server, from where it can be accessed and analysed. Our motivation in this paper is to compare the implementation of health monitoring wireless sensor network architectures based on two popular wireless technologies (Wi-Fi and ZigBee) and analyse the suitability of these technologies for different medical applications.

\section{SySTEM ARCHITECTURES FOR HEALTH MONITORING}

In this section, we discuss the implementation of two architectures for remote monitoring of bio-medical signals. Medical applications have certain nature and requirements that usually have life or death consequences when data is not successfully transferred (e.g. lost, corrupted, delayed, etc.) as opposed to most other applications where requirements and concerns are mostly financial. These requirements such as data rate and delay have been defined by the IEEE 1073 group. For example, in case of 3-lead ECG system, a patient node (i.e., a wireless electrode) generates $2.4 \mathrm{Kbits} / \mathrm{s}$ of data [6]. In our implementations, the sensors used to collect medical data include Blood Pressure, Heart Rate, Temperature, Respiration, Glucose, $\mathrm{SpO} 2$, and ECG. Data rate for bio-medical signal varies significantly. The data rates of various signals are presented in Table 1.

Table 1: Data rate of various bio-medical signals

\begin{tabular}{|c|c|c|}
\hline Bio-medical Signal & Latency & Data Rate \\
\hline Blood pressure & $<3 \mathrm{~s}$ & $80-800 \mathrm{bps}$ \\
\hline Pulse / Heart Rate & $<3 \mathrm{~s}$ & $80-800 \mathrm{bps}$ \\
\hline Glucose & $<3 \mathrm{~s}$ & $80-800 \mathrm{bps}$ \\
\hline Temperature & $<3 \mathrm{~s}$ & $80-800 \mathrm{bps}$ \\
\hline Respiration & $<300 \mathrm{~ms}$ & $50-120 \mathrm{bps}$ \\
\hline SpO2 & $<300 \mathrm{~ms}$ & $50-120 \mathrm{bps}$ \\
\hline ECG & $<300 \mathrm{~ms}$ & $\begin{array}{c}\text { 3-lead }(2.4 \mathrm{kbps}), 5-\mathrm{lead}(10 \\
\mathrm{kbps}), 12-\mathrm{lead}(72 \mathrm{kbps}),\end{array}$ \\
\hline
\end{tabular}

The first architecture implements wireless sensor network based on low-power ZigBee, while the second architecture implements IP-based wireless sensor network using Wi-Fi.

\section{A. ZigBee-Based Architecture}

ZigBee is based on low-rate IEEE 802.15.4 standard, designed for supporting low-power, low-cost, and low-data rate applications. The ZigBee based architecture consists of several patient nodes and a sink node. The system is implemented with

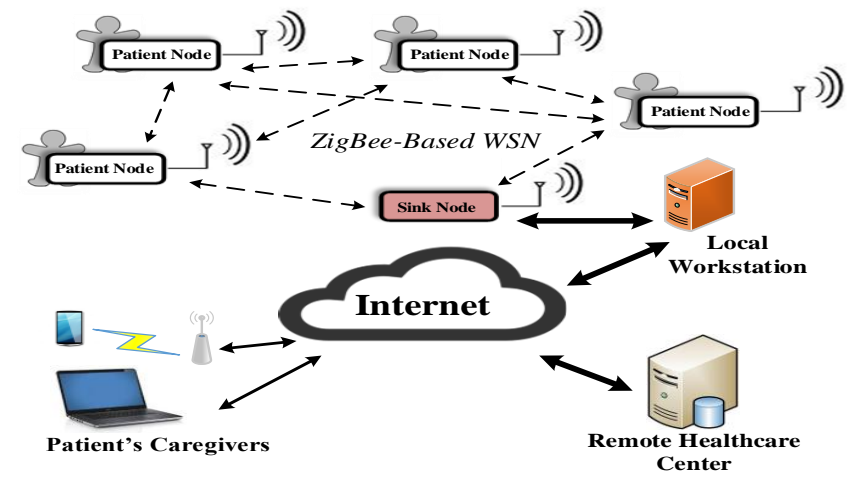

Figure 1: ZigBee Based Health Monitoring System
ZigduinoR2 [7] hardware platform, which is an Arduino compatible microcontroller platform (ATmega128RFA1). contiki operating system is used to implement WSN. ZigBee based architecture as shown in Figure 1 can be divided into four sections; sensor interface, WSN implementation, database application and webserver application.

Sensor interface: The sensor interface is implemented using an Arduino-compatible E-health shield on top of the Zigduino hardware. The E-health shield is basically a gateway between the medical sensors and Zigduino board. Data measured from various sensors are collected by the Zigduino board via the E-health shield.

WSN implementation: The Zigduino's microcontroller contains an on-chip 2.4 GHz IEEE 802.15.4 radio. The implemented WSN consists of several patient (client) nodes and a sink (server) node. Patient nodes collect data from various sensors and send wirelessly over ZigBee to the sink (server) node. The code architecture of sink and patient nodes are shown in Table 2.

Table 2: Code architecture of sink and patient node

\begin{tabular}{|c|c|}
\hline \multicolumn{2}{|c|}{ Server (sink) node architecture } \\
\hline ZigBeeServer & Send and receive data over ZigBee \\
\hline ServiceServer & $\begin{array}{l}\text { Add and remove nodes in the network and assign } \\
\text { ID to them }\end{array}$ \\
\hline MACServer & Grants permission to the nodes to access media. \\
\hline \multicolumn{2}{|c|}{ Client (Patient) node architecture } \\
\hline ZigBeeServer & Send and receive data over ZigBee \\
\hline MeasurementServer & Collect data and store them in FIFO \\
\hline ServiceServer & $\begin{array}{l}\text { Add and remove nodes in the network and assign } \\
\text { ID to them }\end{array}$ \\
\hline MACServer & Grants permission to the nodes to access media \\
\hline
\end{tabular}

Database application: The sink (server) node is connected to a local PC (Personal computer) where a Python code executes to collect data from the serial terminal and save it into a remote database.

Webserver Application: Web-server application written with PHP accesses the database and updates the web page in real time. The data from the webpage can be accessed remotely by patient's caregivers through their laptops or smart phones using any browser.

\section{B. Wi-Fi-Based Architecture}

The Wi-Fi based architecture consists of Wi-Fi enabled sensor nodes (Patient node) to access patient's medical data and Wi-Fi access point (Wi-Fi router). The sensor nodes (Patient node) are designed using an Analog Front-End (AFE, ADS1192 from Texas Instruments, [8]) and Wi-Fi module (RTX4140 Wi-Fi module, [9]). The RTX module is provided with proprietary operating system (ROS). Processor used in the $\mathrm{Wi}-\mathrm{Fi}$ module is EFM32GG230F1024. The architecture (Figure 2) can be divided into four sections; sensor interface, WSN implementation, database application and webserver application.

Sensor interface: The sensor interface is implemented using the AFE to read data from the medical sensors and perform analog to digital conversion. The digital data from the output of AFE is read by RTX4140 through SPI (Serial Peripheral Interface). 
WSN implementation: A UDP (User Datagram Protocol) client application running on the RTX4140 sends the UDP data packet to a remote server through $\mathrm{Wi}-\mathrm{Fi}$, once the connection to the $\mathrm{Wi}-\mathrm{Fi}$ access point is established.

Database application: A UDP server application (running on a remote system), written in python, continuously listens to the UDP port, collects the incoming data and updates a remote database.

Webserver application: Webserver application is same as that of the ZigBee-based architecture.

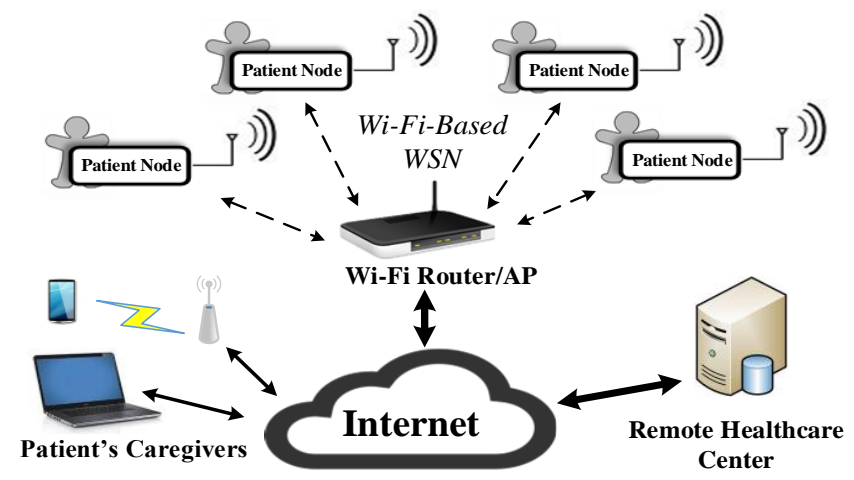

Figure 2: Wi-Fi Based Health Monitoring System

Figure 3 shows the implemented WSN. The patient (client) node collects medical (ECG) data from patient and transmits to the sink (server) node over ZigBee. The sink (server) node is connected to a local PC (Personal computer). The webserver application displays the ECG data on the webpage. In Figure 3, the ECG graph is displayed on a local PC, but it can be accessed from any remote location.

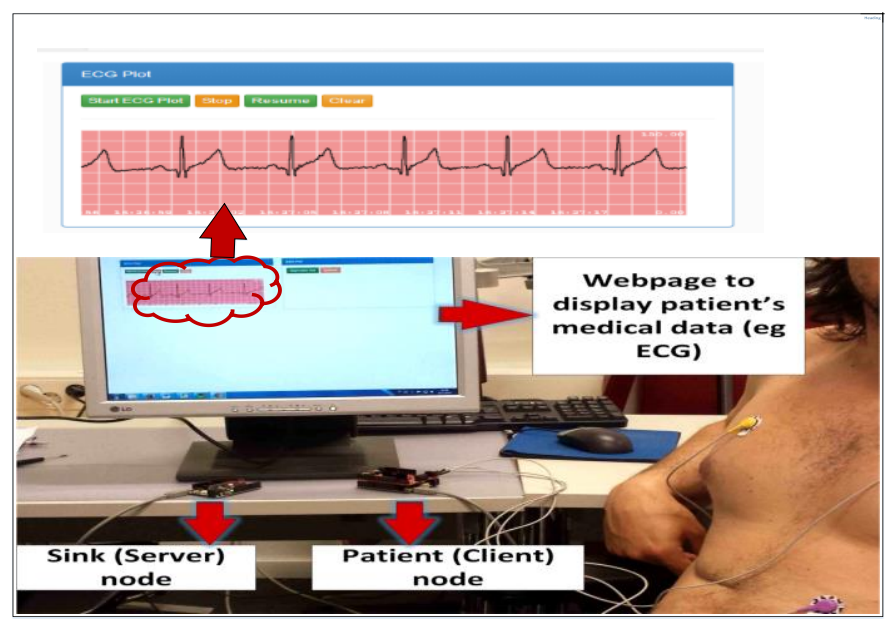

Figure 3: Implementation of WSN

\section{Comparison}

Both of the communication technologies, Wi-Fi and ZigBee, have their advantages and drawbacks. In this section, we discuss some features that influence the selection of the communication technology in the context of healthcare. The features that we will consider are interference, security, energy consumption, reliability, and issue of coexistence. In the following, we further elaborate these features. Table 3 presents a comparison between the two technologies.
ZigBee uses mesh topology which has several advantages over point to point networks in terms of reliability, scalability, and addressing interference issue by virtue of their structure. Reliability in case of Wi-Fi can be addressed with overlapping WAPs (Wireless access points). The mesh topology can scale to hundreds of client nodes easily, but in case of point to point network in order to add an extra client node above 255, an extra access points or router needs to be added [10]. The interference issue in case of mesh can be resolved by choosing an alternate (or best) path [11], whereas in case of point to point networks, it is either required to lower the data rate, lower the transmit power, or change the channel [12]. In order to address the issue of coexistence between ZigBee and $\mathrm{Wi}-\mathrm{Fi}$, dynamic frequency selection and transmission power control is used [13]. Wi-Fi being IP based network provides all the benefits of IP standard such as heterogeneity, compatibility, flexibility, speed, security, efficiency, and accuracy. Power consumption is a concern in case of Wi-Fi with battery life usually ranging from 0.5 to 5 days, whereas in case of ZigBee the battery life can be as long as 1000 days depending upon the application [14]. For security both the technologies use encryption and authentication mechanism; ZigBee uses AES (Advanced Encryption Standard) block cipher with counter mode (CTR), whereas Wi-Fi uses RC4 stream cipher for data encryption. In case of Wi-Fi in order to overcome the weakness of WEP (Wire equivalent privacy), Wi-Fi protected access 2 (WPA2) is used.

Table 3: Comparison between ZigBee and Wi-Fi

\begin{tabular}{|c|c|c|}
\hline Standard & ZigBee & Wi-Fi \\
\hline IEEE spec. & 802.15 .4 & $802.11 \mathrm{a} / \mathrm{b} / \mathrm{g}$ \\
\hline Frequency band & $\begin{array}{c}868 / 915 \mathrm{MHz} ; \\
2.4 \mathrm{GHz}\end{array}$ & $2.4 \mathrm{GHz} ; 5 \mathrm{GHz}$ \\
\hline Max signal rate & $250 \mathrm{~Kb} / \mathrm{s}$ & $54 \mathrm{Mb} / \mathrm{s}$ \\
\hline Nominal range & $10-100 \mathrm{~m}$ & $100 \mathrm{~m}$ \\
\hline Number of RF channels & $1 / 10 ; 16$ & $14(2.4 \mathrm{GHz})$ \\
\hline Channel bandwidth & $\begin{array}{c}0.3 / 0.6 \mathrm{MHz} ; 2 \\
\mathrm{MHz}\end{array}$ & $22 \mathrm{MHz}$ \\
\hline Coexistence mechanism & $\begin{array}{c}\text { Dynamic freq. } \\
\text { selection }\end{array}$ & $\begin{array}{c}\text { Dynamic freq. } \\
\text { selection, transmit } \\
\text { power control }\end{array}$ \\
\hline Battery Life (days) & $100-1,000$ & $0.5-5.0$ \\
\hline Basic cell & Star & $\begin{array}{c}\text { BSS (basic service } \\
\text { set) }\end{array}$ \\
\hline Extension of the basic cell & Cluster tree, & $\begin{array}{c}\text { ESS (extended } \\
\text { service set) }\end{array}$ \\
\hline Max number of cell nodes & $>65000$ & 255 \\
\hline Encryption & AES block & $\begin{array}{c}\text { RC4 stream cipher } \\
\text { (WEP), AES block } \\
\text { cipher (CTR, }\end{array}$ \\
\hline
\end{tabular}

IV. DEMONSTRATORS, RESULTS AND DisCUSSION

The experimental setup to compare both the architectures is shown in Figure 4. The scenario consists of a hospital room with twenty patient nodes reading patient's medical data from various sensors including 2-lead ECG, SpO2, Blood Pressure, Heart Rate, Temperature, Respiration, and Glucose level. There is one sink node (for ZigBee based architecture) or a Wi-Fi access point (for Wi-Fi based architecture) to collect data from all the patient nodes in their respective setup. The distance between the adjacent patient nodes in same column is two meters and the distance between the adjacent patient nodes in different column is six meters. Every patient node transmits about 8.7 kbits (payload) of data per second. Figure 5 summarizes the average power consumption $(\mathrm{mW})$ by the 
patient (client) nodes of Wi-Fi and ZigBee based architectures, with respect to the experimental setup discussed.

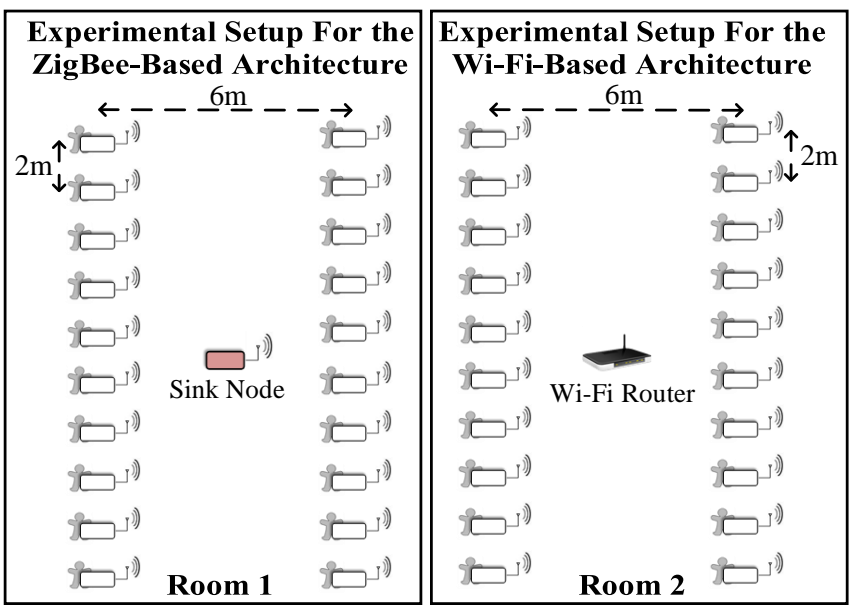

Figure 4: Experimental setup to compare both the architectures

The power consumption in case of three different Wi-Fi protocols $802.11 \mathrm{~b} / \mathrm{g} / \mathrm{n}$ are $14,17.5$, and $14 \mathrm{~mW}$ respectively, whereas in case of the ZigBee based network the power consumption is considerably less $(2.4 \mathrm{~mW})$.

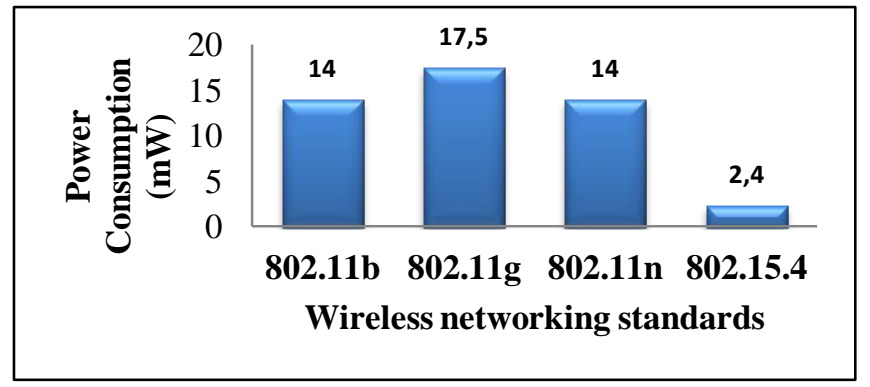

Figure 5: Average power consumption in ZigBee and Wi-Fi based sensor nodes

Thus we can observe that the power consumption in ZigBee based network is almost 6 to 7 times less ( 7 times for $802.11 \mathrm{~g}$ and 6 times for $802.11 \mathrm{~b} / \mathrm{n}$ ) when compared with Wi-Fi based network for the same experimental setup. At this point it is worth noting that although Wi-Fi based network consumes more power than ZigBee for lower data-rate, with increase in data rate, power consumption in ZigBee increases rapidly when compared to $\mathrm{Wi}$ Fi. In practise the maximum data-rate achieved for transmitting sensor data with ZigBee using contiki OS is $160 \mathrm{Kbits} / \mathrm{sec}$, when the nodes are placed at a distance of around 10 meters. In case of star topology the network can support up to 18 nodes, whereas in case of mesh topology using multi-hopping each nodes can route data of up to 17 other nodes apart from transmitting the data acquired, thus increasing the scalability to higher number. At the present data rate $(8.7 \mathrm{kbits} / \mathrm{sec}$ payload) required, scalability is not an issue in case of $\mathrm{Wi}-\mathrm{Fi}$ and the system can be scaled to large number of nodes using single access-point.

\section{CONCLUSIONS}

In this paper, we presented wireless systems for remote monitoring of bio-medical signals to alleviate issues in traditional health monitoring systems and to improve the quality of medical care. Two variants of the wireless health monitoring systems are implemented to remotely monitor patients. One system implements wireless sensor network based on low power ZigBee. The system consists of set of sensor nodes (clients) to read data from various medical sensors process it and send wirelessly over ZigBee to a server node. The other system implements IP-based wireless sensor network, using Wi-Fi. The system consists of WiFi based sensor module to access bio-medical signals from patients and send it to a remote server which updates the database in realtime. In both implementations, the server node collects the medical data from several client nodes and updates a remote database. The webserver application accesses the database and updates the webpage in real-time, which can be accessed remotely.

\section{REFERENCES}

[1] D. Evans, "The Internet of Things How the Next Evolution of the Internet Is Changing Everything", "Cisco Internet Business Solutions Group (IBSG)", white paper, 2011, retrieved on May 2, 2014 from https://www.cisco.com/web/about/ac79/docs/innov/IoT_IBSG_0411FIN AL.pdf

[2] L. Piccini et al., "Wireless DSP architecture for biosignals recording", December 2004, Volume 18 Issue 21 pages 487-490

[3] Huimin She et ai., "A Network-based System Architecture for Remote Medical Applications", Dept. of Electronic, Computer and Software Systems, Royal Institute of Technology, Sweden, ASIC \& System State Key Lab., Dept. of Microelectronics, Fudan Univ., Shanghai, China, 2007.

[4] Benny P.L. Lo et al., "Body sensor network - A wireless sensor platform for pervasive healthcare monitoring", Adjunct Proceedings of the 3rd International conference on Pervasive Computing (PERVASIVE'05), May 2005, pages 77-80

[5] R.S .H. Istepanian et al., "The Potential of Internet of m-health Things "m-IoT" for Non-Invasive Glucose level Sensing". In proceeding of IEEE, 2011 pages 5264-5266

[6] Christos Tachtatzis et al., "An Energy Analysis of IEEE 802.15.6 Scheduled Access Modes for Medical Applications", D. Simplot-Ryl et al. (Eds.): ADHOCNETS 2011, LNICST 89, pp. 209-222.

[7] Zigduino r2, retrieved on Jul 172014 from http://www.logoselectro.com/store/zigduino-r2

[8] ADS1192 Demonstration Kit, retrieved on Jul 172014 from http://www.ti.com/tool/ads1192ecg-fe

[9] RTX41xx Low Power Modules, retrieved on Jul 172014 from http://www.rtx.dk/RTX41xx_Modules-4024.aspx

[10] Wireless Connectivity for Medical Applications, retrieved on June 12 2014, from http://www.arrownac.com/eventstraining/training/pdfs/wireless.pdf

[11] ZigBee and Wireless Radio Frequency Coexistence, retrieved on Jun 12 2014 from https://docs.zigbee.org/zigbee-docs/dcn/07-5219.PDF

[12] Coping with Wi-Fi's biggest problem: interference, retrieved on Jun 12 2014 from http://www.networkworld.com/article/2215287/techprimers/coping-with-wi-fi-s-biggest-problem--interference.html

[13] Jin-Shyan Lee et al., "A Comparative Study of Wireless Protocols: Bluetooth, UWB, ZigBee, and Wi-Fi", In proceeding Industrial Electronics Society, 2007. IECON, IEEE, 2007, Pages 46-51

[14] Kartik Rathod et al., "Wireless automation using ZigBee protocols ", published in Wireless and Optical Communications Networks (WOCN),2012,pages 1-5

[15] D. Miorandi et al., "Internet of things: Vision, applications and research challenges", Ad Hoc Networks, Sep 2012, Volume 10 Issue 7, pages 1497-1516 